THIS volume* contains 18 papers presented in 1972 at a conference attended by scientists, historians and philosophers at the Villa Serbonelloni in Bellagio, Italy. Each paper is followed by comments of one or more of the participants, usually Bernard Rensch. If the subject of this conference was, as Ledyard Stebbins ( $p$. 285) claims, reduction in biology, then few of the participants addressed themselves to the issue.

The two boundaries between natural phenomena which are of greatest interest in arguments over reduction are that between the mind and physiological processes and that between biological processes in general and the subject matter of physics and chemistry. Until quite recently, all of the carefully worked out examples of reduction have concerned reduction within physics, the reduction of one physical theory to another. Thus, the next step would seem to be the anlaysis of similar examples within biology. Furthermore, these are exactly the examples that professional biologists are best prepared to deal with constructively. But the topic never arose. Instead, most of the papers which touched on reduction at all, dealt with the reduction of mind to physiological processes and a few concerned the reduction of biological processes to physics and chemistry.

The papers divide fairly naturally into three groups: historical, biological, and philosophical. The philosophical papers divide somewhat less easily into those which are straightforward philosophy and those which are 'philosophical' in the pejorative sense. The papers by Ernest Boesinger, June Goodfield, and G. Montalenti are primarily historical. Boesinger traces the fate of the evolutionary hypothesis after Lamarck and Darwin, giving special attention to the bizarre situation which still prevails in France. Goodfield attempts to derive some conclusions about reductive strategies by studying the methods and results of a variety of biologists in the 19th and 20th centuries. Montalenti compares mechanistic reductionism and holistic vitalism in Democritus, Aristotle, and Darwin. Gerald Edelman's paper is also historical to some extent, discussing as he does the recent history of the theory of clonal

* Studies in the Philosophy of Biology: Reduction and Related Problems. Edited by Francisco J. Ayala and Theodosius Dobzhansky. Pp xix +390 . (Macmillan: London and Basingstoke, September 1974.) $£ 12.00$.

\section{Philosophy of reductionism in biology}

Aristotle-mechanistic reductionism and holistic vitalism. Taken from Hartmann

Schedel Liber Chronicarium (Nuremberg Chronicle), Nurnberg, 1493.

selection in antibody formation. The papers by Dobzhansky, Stebbins and John C. Eccles are mainly biological. If there was ever an excuse for the illinformed parodies that so often pass for discussions of the synthetic theory of evolution, Dobzhansky's lucid synopsis eliminates it once and for all.

The papers by Morton Beckner, Dudley Shapere, Francisco Ayala, Donald Campbell, and Karl Popper are truly philosophical. Beckner exploits the distinction between hierarchically arranged theories and hierarchically organised natural systems. Shapere expands upon the difference between compositional and evolutionary theories and the different sorts of problems which give rise to them. Donald Campbell sets out his views on evolutionary epistemology, views shared in part by Popper and Henryk Skolimowski. Just as biological species adapt to their changing environments by a process of variation and selective retention, individual organisms come to learn about their environments and successive scientific theories come to characterise the real world with ever increasing accuracy. One problem with this philosophical thesis concerns the appropriate adjective required to modify the term 'variation'. In what sense are mutations 'chance'? In what sense is learning and the development of science 'blind'?

Ayala presents a surprisingly informative and sensitive linguistic analysis of "evolutionary progress", surprising because such analyses are rarely informative and even more rarely produced by practicing scientists. Stebbins also deals with the notion of progress in his contribution. Peter Medawar presents an uncontroversial comparison between levels of generality in geometry and the natural sciences. Skolimowski, on the other hand, presents an unnecessarily petulant attack on the conventional notion of rationality in science. The tone of Skolimowski's paper is especially unfortunate because the issues are both important and inherently liable to distortion and caricature.

Large chunks of the papers by Rensch, Eccles, W. H. Thorpe, and Charles Birch exemplify what can happen when a noted scientist tries his hand at 'philosophising': these papers are uncomfortable reading. The biology is accurate and often inherently fascinating - for example, Eccles' discussion of split brain experiments and Thorpe's description of migration and exploratory learning-but too often their attempts to set out philosophical theses are as embarrassing as the efforts of an ageing diva trying to sing the latest pop song. Although Shapere values interdisciplinary endeavours, he replies to these poorly formulated views in the only way a professional philosopher can (pp. 256,258 ). Just as scientists are entitled to establish standards of competence for their undertakings, philosophers have a right to expect at least minimal competence in theirs.

I have, however, saved the best part of the book until last. Monod did not present a formal paper at the conference; instead, he defended his book Chance and Necessity. The ensuing discussion points up, in the most direct manner possible, both the obscurity and importance of the problems which surround the issue of reduction. Several of the papers in this volume go a little way towards reducing the obscurity, and the emotional exchange between Monod and Skolimowski attests to the importance of these issues. I could not recommend that anyone read this volume cover to cover, but a few of the papers are worthwhile and the final 20 pages, in which the participants quiz Monod, should not be missed.

David L. Hull 